\title{
Association of Age and Anemia With Adiponectin Serum Levels in Normal-Weight Japanese Women
}

\author{
Mari Honda ${ }^{\mathrm{a}, \mathrm{b}}$, Ayaka Tsuboic, d, Satomi Minato ${ }^{\mathrm{c}, \mathrm{e}}$, Kaori Kitaoka ${ }^{\mathrm{c}, \mathrm{f}}$, Mika Takeuchic, g, \\ Megumu Yano ${ }^{\text {h, Miki Kurata }}{ }^{\mathrm{c}, \mathrm{h}}$, Bin Wu $\mathrm{W}^{\mathrm{a}, \mathrm{i}}$, Tsutomu Kazumia, c, j, k, \\ Keisuke Fukuo ${ }^{\mathrm{a}, \mathrm{c}, \mathrm{h}}$
}

\begin{abstract}
Background: Adiponectin serum levels are affected by sex, ethnicities, adiposity, age and several pathological conditions such as anemia. The prevalence of hyperadiponectinemia $(\geq 20 \mathrm{mg} / \mathrm{L})$ in relation to anemia (hemoglobin $<12 \mathrm{~g} / \mathrm{dL}$ ) was examined in normal-weight Japanese women.
\end{abstract}

Methods: Serum adiponectin and blood hemoglobin were measured in 311 young women aged 18 - 24 years (A), 148 of their middleaged mothers aged $39-60$ years (B) and 322 community-dwelling women aged $\geq 65$ years $(\mathrm{C})$ with a mean body mass index (BMI) of $20.4,22.0$ and $22.4 \mathrm{~kg} / \mathrm{m}^{2}$, respectively. Elderly women were subdivided into three age groups: between 65 and 74 years $(n=95, X)$, between 75 and 84 years $(n=176, Y)$ and older than 85 years $(n=$ $51, \mathrm{Z})$.

Results: The prevalence of hyperadiponectinemia (A: 3.9\%, B: $3.4 \%$, $\mathrm{C}: 22.7 \%, \mathrm{P}<0.001$ ) was low and serum adiponectin (A: $11.5 \pm 4.3$

Manuscript submitted March 18, 2019, accepted April 1, 2019

${ }^{a}$ Open Research Center for Studying of Lifestyle-Related Diseases, Mukogawa Women's University, Nishinomiya, Hyogo, Japan

bDepartment of Health, Sports, and Nutrition, Faculty of Health and Welfare, Kobe Women's University, Kobe, Hyogo, Japan

'Research Institute for Nutrition Sciences, Mukogawa Women's University, Nishinomiya, Hyogo, Japan

${ }^{\mathrm{d} D e p a r t m e n t}$ of Nutrition, Osaka City Juso Hospital, Osaka, Japan

${ }^{\mathrm{e}}$ Graduate School of Human Science and Environment, University of Hyogo, Himeji, Hyogo, Japan

${ }^{f}$ Department of Nutritional Sciences for Well-being, Faculty of Health Sciences for Welfare, Kansai University of Welfare Sciences, Kashiwara, Osaka, Japan

gDepartment of Food Sciences and Nutrition, Faculty of Human Life and Environmental Sciences, Nagoya Women's University, Nagoya, Aichi, Japan hDepartment of Food Sciences and Nutrition, School of Human Environmental Sciences, Mukogawa Women's University, Nishinomiya, Hyogo, Japan iDepartment of Endocrinology, First Affiliated Hospital of Kunming Medical University, Kunming, Yunnan, China

jDepartment of Medicine, Kohnan Kakogawa Hospital, Kakogawa, Hyogo, Japan

${ }^{\mathrm{k}}$ Corresponding Author: Tsutomu Kazumi, Research Institute for Nutrition Sciences, Mukogawa Women's University, 6-46, Ikebiraki-cho, Nishinomiya, Hyogo 663-8558, Japan. Email: kazumi@mukogawa-u.ac.jp

doi: https://doi.org/10.14740/jocmr3821 $\mathrm{mg} / \mathrm{L}, \mathrm{B}: 11.8 \pm 4.9 \mathrm{mg} / \mathrm{L}, \mathrm{C}: 15.3 \pm 7.8 \mathrm{mg} / \mathrm{L}, \mathrm{P}<0.001)$ did not change until middle-aged but increased thereafter in a stepwise fashion (X: 18.9\%, Y: 22.7\%, Z: 35.3\%, P = 0.07 and $X: 13.9 \pm 6.9 \mathrm{mg} / \mathrm{L}$, $\mathrm{Y}: 15.1 \pm 7.7 \mathrm{mg} / \mathrm{L}, \mathrm{Z}: 18.7 \pm 8.6 \mathrm{mg} / \mathrm{L}, \mathrm{P}=0.001$, respectively). There were inverse associations of adiponectin with age $(\mathrm{r}=-0.201, \mathrm{P}$ $<0.001)$ and hemoglobin $(\mathrm{r}=-0.318, \mathrm{P}<0.001)$ in elderly women but not even in young and middle-aged women combined. Furthermore, anemia was associated with higher prevalence of hyperadiponectinemia $(34.8 \%$ vs. $20.6 \%, \mathrm{P}=0.01)$ and higher serum adiponectin $(18.3$ $\pm 9.4 \mathrm{mg} / \mathrm{L}$ vs. $14.5 \pm 7.1 \mathrm{mg} / \mathrm{L}, \mathrm{P}<0.001)$ in elderly women but not in younger and middle-aged women.

Conclusions: In normal-weight Japanese women, the prevalence of hyperadiponectinemia and serum adiponectin were increased and associated with anemia at 65 years of age and older.

Keywords: Adiponectin; Hemoglobin; Anemia; Aging; Women

\section{Introduction}

Adiponectin, an adipocyte-derived peptide, circulates in high concentrations (1,000-fold higher relative to other adipokines) and has been shown to have insulin-sensitizing, anti-inflammatory, anti-atherogenic and anti-apoptotic properties in experimental studied [1]. Racial, ethnic and sex differences exist in serum adiponectin concentrations [2,3], which are decreased in obesity and increased after weight loss $[4,5]$. Although some studies reported that serum adiponectin declined or did not change with age [6-8], others reported elevated serum adiponectin in the elderly [9-13]. This seems to be a contradiction because aging is associated with an increase in abdominal obesity [14].

Studies including ours reported that anemia or low hemoglobin was associated with high circulating adiponectin in community-dwelling people [15-20]. Causes of anemia are different between premenopausal and postmenopausal women. In non-pregnant, premenopausal women, anemia due to menstrual blood loss is common [20]. In the elderly people, anemia of indeterminate cause was present in one-third, while anemia of nutrient deficiency was present in one-third and anemia of chronic inflammation or chronic renal disease or both was present in one-third [21]. However, to our 
Table 1. Anthropometric, Laboratory and Clinical Characteristics of Young, Middle-Aged and Elderly Japanese Women

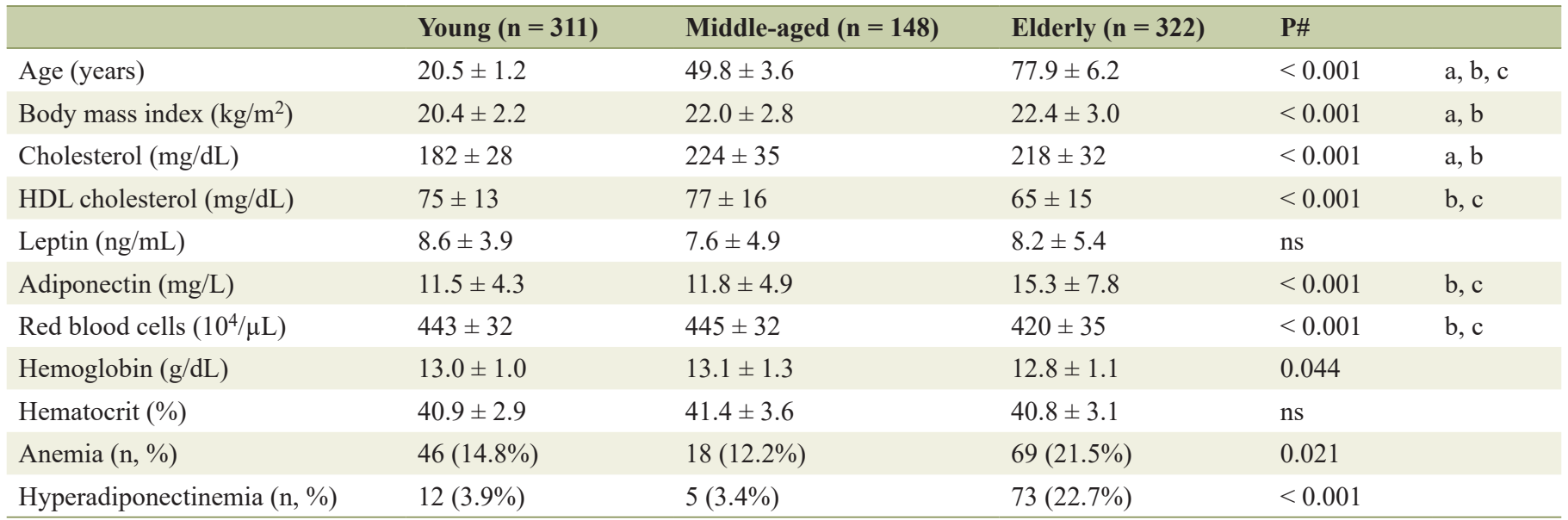

Mean \pm SD or $n, \%$. \#P values by ANOVA or Chi-square test. a, b: Young versus middle-aged and elderly, respectively; c: Middle-aged versus elderly: significantly different at $\mathrm{P}<0.05$ or less by Bonferroni's multiple comparison procedures. HDL: high-density lipoprotein; SD: standard deviation; ANOVA: analysis of variance.

knowledge, there is no report to date to assess the relationship of adiponectin with anthropometric, metabolic and hematological variables from healthy young adults to elderly people. Therefore, we investigated these issues in young, middle-aged and elderly Japanese women who were of normal-weight in the present study.

\section{Patients and Methods}

We studied cross-sectionally 311 young female students of the university aged 18 - 24 years (A), 148 their biological mothers aged 39 - 60 years (B) and 322 community-dwelling women aged $\geq 65$ years $(C)$. Characteristics of three groups of women were described in detail elsewhere [17, 22, 23]. Women in groups $\mathrm{B}$ and $\mathrm{C}$ were referred to as middle-aged and elderly women, respectively. Elderly women were subdivided into three age groups: between 65 and 74 years $(X, n=95)$, between 75 and 84 years $(Y, n=176)$ and between 85 and 96 years $(\mathrm{Z}, \mathrm{n}=51)$. Subjects with clinically diagnosed acute or chronic inflammatory diseases, endocrine, cardiovascular, hepatic, renal diseases, hormonal contraception and unusual dietary habits were excluded from the study. The study was approved by the Mukogawa Women's University Ethical Committee (No. 07-28 on 19/02/2008 and No. 11-7 on 20/5/2011) to be in accordance with the Helsinki Declaration.

Body weight and height were measured, and blood was drawn after an overnight fast in young and middle-aged women and 159 elderly women. The remaining 202 elderly women received anthropometric measurement and blood samplings after breakfast between 9:30 and 10:30 am. Serum cholesterol, high-density lipoprotein (HDL) cholesterol and leptin were measured as previously reported [22, 23]. Adiponectin was assayed by a sandwich enzyme-linked immunosorbent assay (Otsuka Pharmaceutical Co., Ltd, Tokushima City, Japan). Intra- and interassay coefficients of variation (CV) were $3.3 \%$ and $7.5 \%$, respectively. Leptin was assessed by an RIA kit from LINCO research (St. Charles, MO, interassay CV 4.9\%). Complete blood cell count was analyzed using an automated blood cell counter (Sysmex XE-2100, Sysmex, Kobe, Japan). Hyperadiponectinemia was defined as having adiponectin $\geq 20$ $\mathrm{mg} / \mathrm{L}[24,25]$ and anemia was defined as having blood hemoglobin $<12.0 \mathrm{~g} / \mathrm{dL}$ [26].

In elderly women, serum creatinine was measured enzymatically using an auto-analyzer (AU 5200, Olympus, Tokyo, Japan) and the estimated glomerular filtration rate (eGFR) was calculated using the equation recommended by the Japanese Society for Nephrology [27].

Data were presented as mean \pm standard deviation (SD) unless otherwise stated. Differences in frequencies of conditions were analyzed by $\chi^{2}$ test and between two groups by $t$-test. Differences among three groups were analyzed using analysis of variance. When $\mathrm{P}$ values in analysis of variance were $<0.05$, Bonferroni's multiple comparison procedure was performed. Bivariate correlations of adiponectin with anthropometric, clinical and cardiometabolic parameters were evaluated by Pearson correlation analysis. A two-tailed $\mathrm{P}<0.05$ was considered statistically significant. All calculations were performed with SPSS system 15.0 (SPSS Inc., Chicago, IL, USA).

\section{Results}

As shown in Table 1, mean body mass index (BMI) was of normal weight in participants of three age groups, who had normal serum cholesterol and relatively high HDL cholesterol. Middle-aged and elderly women had higher BMI compared with young women. Changes in serum leptin were modest.

The prevalence of hyperadiponectinemia was low (Fig. 1) and serum adiponectin did not differ between young and middle-aged women (Table 1). However, elderly women had higher serum adiponectin (Table 1) and prevalence of hyperadiponectinemia (Fig. 1). The prevalence of anemia was higher in elderly women compared with young and middle-aged women 

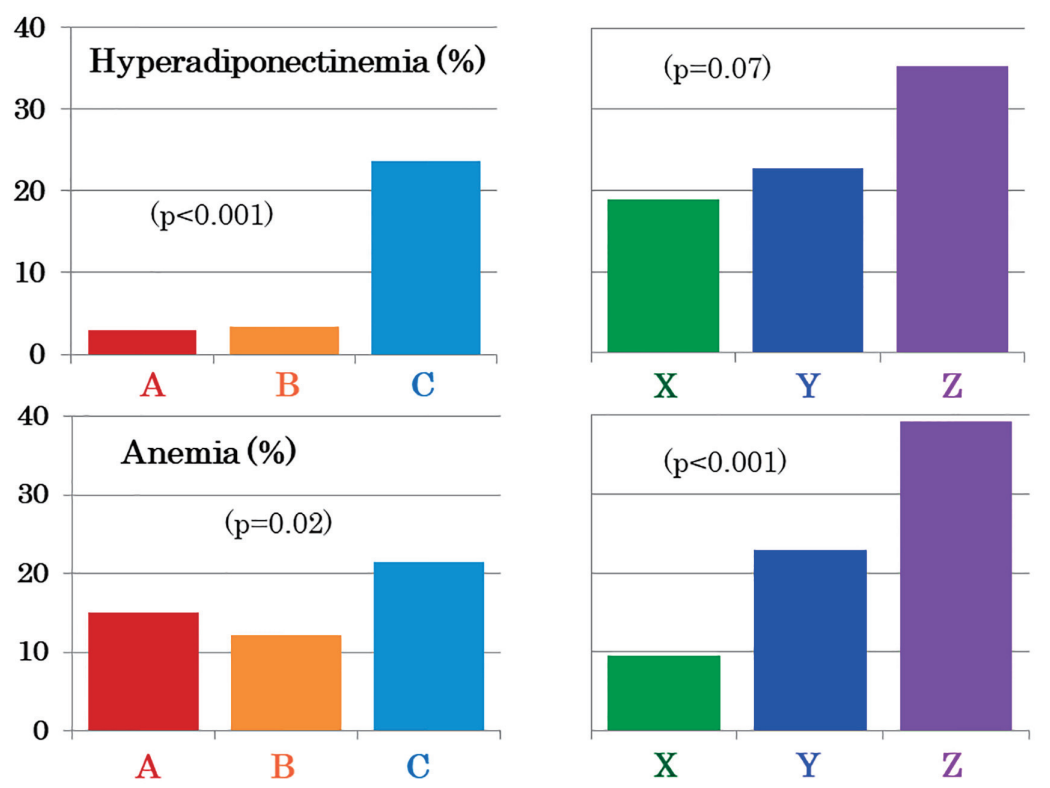

Figure 1. The prevalence of hyperadiponectinemia (upper panel) and anemia (lower panel) in 311 young (A, red bars), 148 middle-aged ( $B$, yellow bars) and 322 elderly women ( $C$, light blue bars). Elderly women were subdivided into three age groups: between 65 and 74 years $(n=95, X$, green bars), between 75 and 84 years $(n=176, Y$, dark blue bars) and between 85 and 96 years $(n=51, Z$, purple bars).

(Fig. 1), although blood hemoglobin did not differ among the three groups (Table 1). There was no difference between 123 premenopausal and 25 postmenopausal middle-aged women in levels of adiponectin and hemoglobin (data not shown) as well as the prevalence of hyperadiponectinemia $(3.3 \%$ vs. $4.0 \%, \mathrm{P}$ $=0.8)$ and anemia $(12.3 \%$ vs. $12.0 \%)$.

Among elderly women, the prevalence of hyperadiponectinemia (Fig. 1) and serum adiponectin (Table 2) increased in a stepwise fashion with advancing age. Adiponectin was higher in women aged $\geq 85$ years than the other two groups. The prevalence of anemia increased (Fig. 1), and blood hemoglobin decreased in a stepwise fashion (Table 2). There also was a stepwise decrease in eGFR with advancing age (Table 2). After controlling for hemoglobin, a difference in serum adiponectin was no longer significant, whereas it was still highly significant, though attenuated, after controlling for eGFR (data not shown). After controlling for both hemoglobin and eGFR, a difference in serum adiponectin was no longer significant $(\mathrm{X}$ : $14.9 \pm 0.8 \mathrm{mg} / \mathrm{L}, \mathrm{Y}: 14.9 \pm 0.6 \mathrm{mg} / \mathrm{L}, \mathrm{Z}: 17.5 \pm 1.1 \mathrm{mg} / \mathrm{L}, \mathrm{P}=$ 0.098 , mean \pm standard error of the mean (SEM)).

Elderly women with anemia $(n=69)$ had higher serum adiponectin (Fig. 2) and higher prevalence of hyperadiponectinemia $(34.8 \%$ vs. $20.6 \%, \mathrm{P}=0.01)$ compared to 252 elderly women without anemia. In contrast, young and middleaged women showed no intra-group difference in adiponectin between the presence and absence of anemia (Fig. 2). Hemoglobin did not differ among three age groups of women with anemia (Fig. 2). As compared to young and middle-aged women, elderly women who did not have anemia still had elevated serum adiponectin $(14.5 \pm 7.1 \mathrm{mg} / \mathrm{L}, \mathrm{P}<0.01)$ and higher prevalence of hyperadiponectinemia $(20.6 \%, \mathrm{P}<0.01)$.

As shown in Table 3, adiponectin showed a positive as- sociation with age and inverse associations with hemoglobin (Fig. 3), red blood cells and hematocrit in elderly women but not in young and middle-aged women. To obtain a wider range of age (18 - 60 years), young and middle-aged women were combined and re-analyzed. Again, there was no association between adiponectin and age (Fig. 4) and between adiponectin and hemoglobin $(r=0.03, P=0.6)$. Inverse association of adiponectin with BMI and positive association with HDL cholesterol were evident among three age groups (Table 3 ).

Elderly women with compared to those without hyperadiponectinemia were older and had lower BMI and serum leptin, whereas serum cholesterol did not differ (Table 4). They had higher HDL cholesterol. Furthermore, they had lower red blood cells, hemoglobin and hematocrit and hence higher prevalence of anemia.

\section{Discussion}

To our knowledge, this is the first study to date to assess the relationship of adiponectin with anthropometric, metabolic and hematological variables in healthy normal-weight women with a broad range of age (18 - 96 years). Our study demonstrates that serum adiponectin and the prevalence of hyperadiponectinemia in fact increased at 65 years of age and older, whereas the prevalence of hyperadiponectinemia was low and serum adiponectin did not change with age until middle-aged. These findings are supported by observations that adiponectin showed no correlation with age when young and middleaged women were combined and re-analyzed to obtain a wider age range. Inverse association between adiponectin and age was evident in elderly women. In addition, the current study 
Table 2. Features of Elderly Women Divided Into Three Age Groups

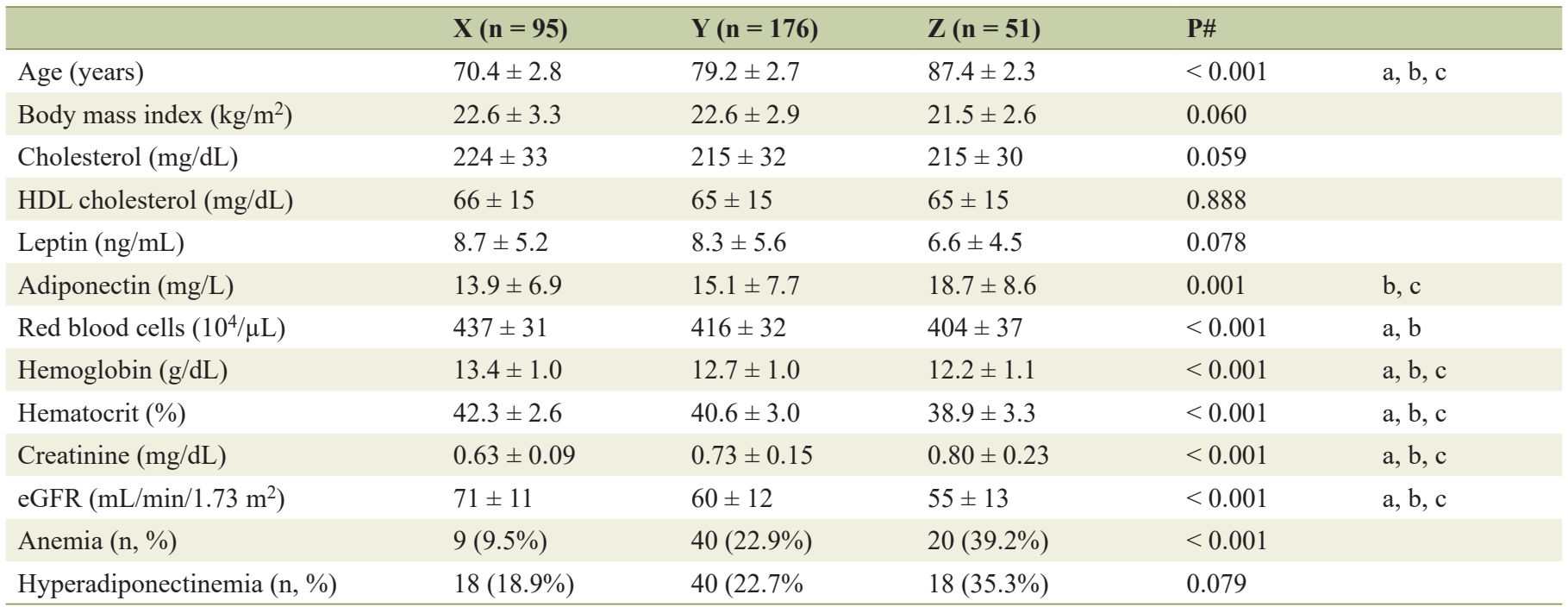

Mean \pm SD or $n, \%$. \#P values by ANOVA or Chi-square test. a, b: Young versus middle-aged and elderly, respectively; c: Middle-aged versus elderly: significantly different at $\mathrm{P}<0.05$ or less by Bonferroni's multiple comparison procedures. X: Between 65 and 74 years; $Y$ : Between 75 and 84 years; Z: Between 85 and 96 years. eGFR: estimated glomerular filtration rate; HDL: high-density lipoprotein; SD: standard deviation; ANOVA: analysis of variance.

highlights negative correlation between adiponectin and hemoglobin in elderly but not in young and middle-aged women. Anemia was associated with hyperadiponectinemia in elderly women, whereas in young and middle-aged women, the prevalence of hyperadiponectinemia was low and serum adiponectin did not differ between those with and without anemia.

As previously reported [9-13] and confirmed in the present study, healthy elderly subjects had elevated serum adiponectin. In a large-scale Japanese study of people who underwent medical check-up [9], 2,122 women aged $<40$ years and 2,534 women aged 40 - 50 years had a least square mean adiponectin of 10.7 and $11.1 \mathrm{mg} / \mathrm{L}$, respectively. Although the difference is statistically significant, the prevalence of hyperadiponectinemia $(\geq 20 \mathrm{mg} / \mathrm{L})$, which seems to be of clinical significance $[24,25]$, was not reported [9]. In the present study, serum adiponectin did not change in young and middle-aged women. In addition, the prevalence of hyperadiponectinemia was extremely low in both young and middle-aged women. Further-

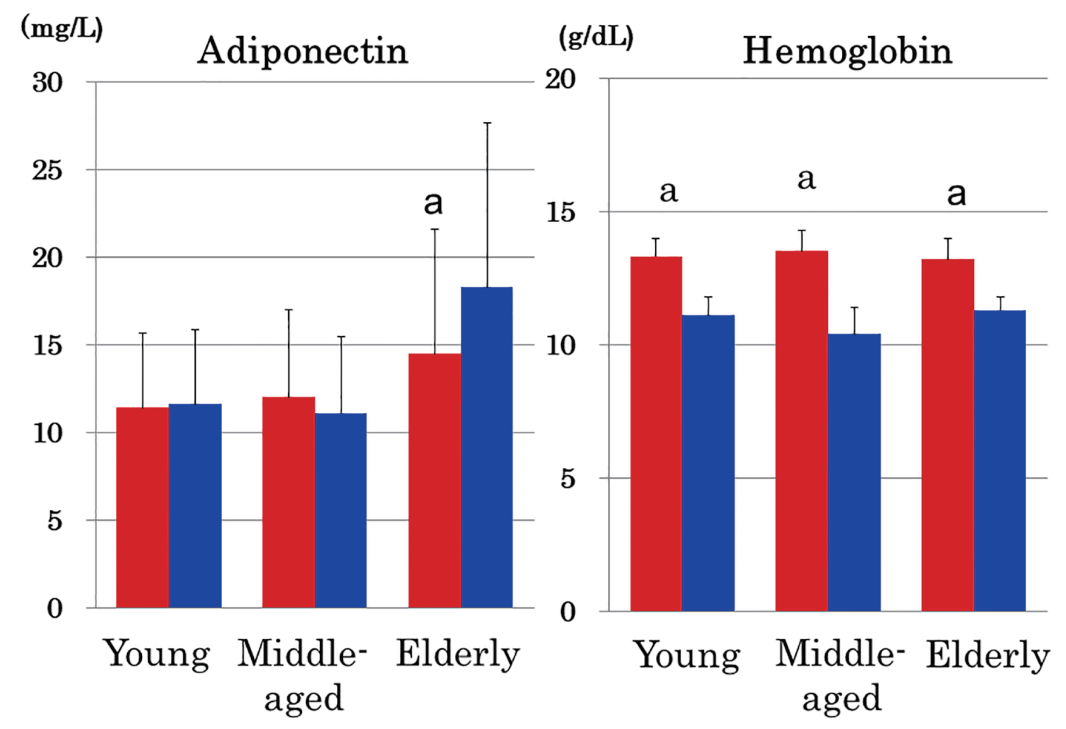

Figure 2. Serum adiponectin and hemoglobin in young, middle-aged and elderly women in the absence (red columns) and presence of anemia (blue columns). Note that adiponectin was elevated in anemic elderly women but not in anemic young and middle-aged women despite the fact that the degree of anemia of elderly women was comparable with that of young and middleaged women. Mean \pm SD. a: $P<0.001$ between women with and without anemia. 
Table 3. Correlation Coefficients of Serum Adiponectin in Young, Middle-Aged and Elderly Japanese Women

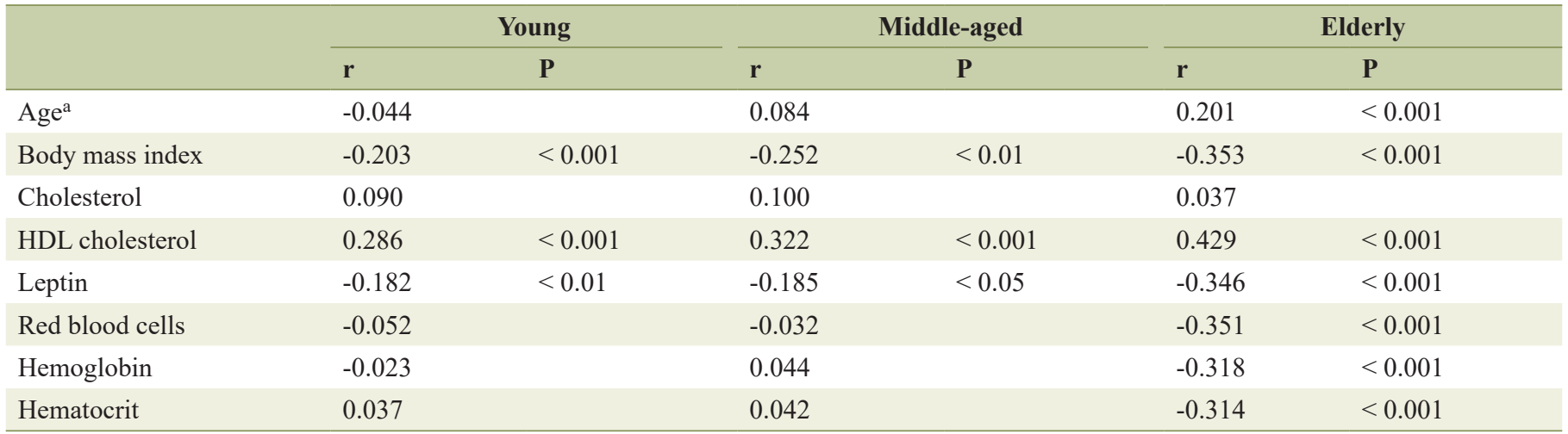

aRanges of age (years): young, 18 - 24 years; middle-aged, 39 - 60 years; elderly, 65 - 96 years. HDL: high-density lipoprotein.

more, there was no association between adiponectin and age $(\mathrm{r}$ $=-0.001, \mathrm{P}=0.9)$ when young and middle-aged women were combined to obtain a wider range of age (18 - 60 years) and reanalyzed. Some observations that serum adiponectin declined or did not change with age [6-8] may be a consequence of a wider range of adiposity and/or a narrow range of age.

Serum adiponectin and the prevalence of hyperadiponectinemia were the highest in women aged $\geq 85$ years $(Z)$ among three groups of elderly women. Interval increases in adiponectin were associated with decreasing body weight in old age [28]. However, BMI did not change with advancing age in our elderly women. Instead, the prevalence of anemia was the highest and hemoglobin was the lowest in women aged $\geq 85$ years among three groups of elderly women.

In the present study, hemoglobin showed no association with adiponectin in young and middle-aged women, in whom anemia due to menstrual blood loss is common [20]. This is in contrast with inverse association between adiponectin and hemoglobin as previously reported in community-dwelling elderly people [15-20] and confirmed in the present study. These findings indicate that elevated adiponectin is not directly related to low hemoglobin or anemia per se but suggest that etiologies of anemia may be related to hyperadiponectinemia in anemic elderly women. Major causes of anemia in elderly people were nutrient deficiency and chronic inflammation and/ or chronic renal disease, and cause was indeterminate in onethird [21].

Reasons why serum adiponectin was associated with anemia and age in elderly but not in young and middle-aged women remain unclear. As bone marrow adipocytes increase in number with age [28] and synthesize adiponectin, which promotes proliferation of hematopoietic stem cells [29], hy-

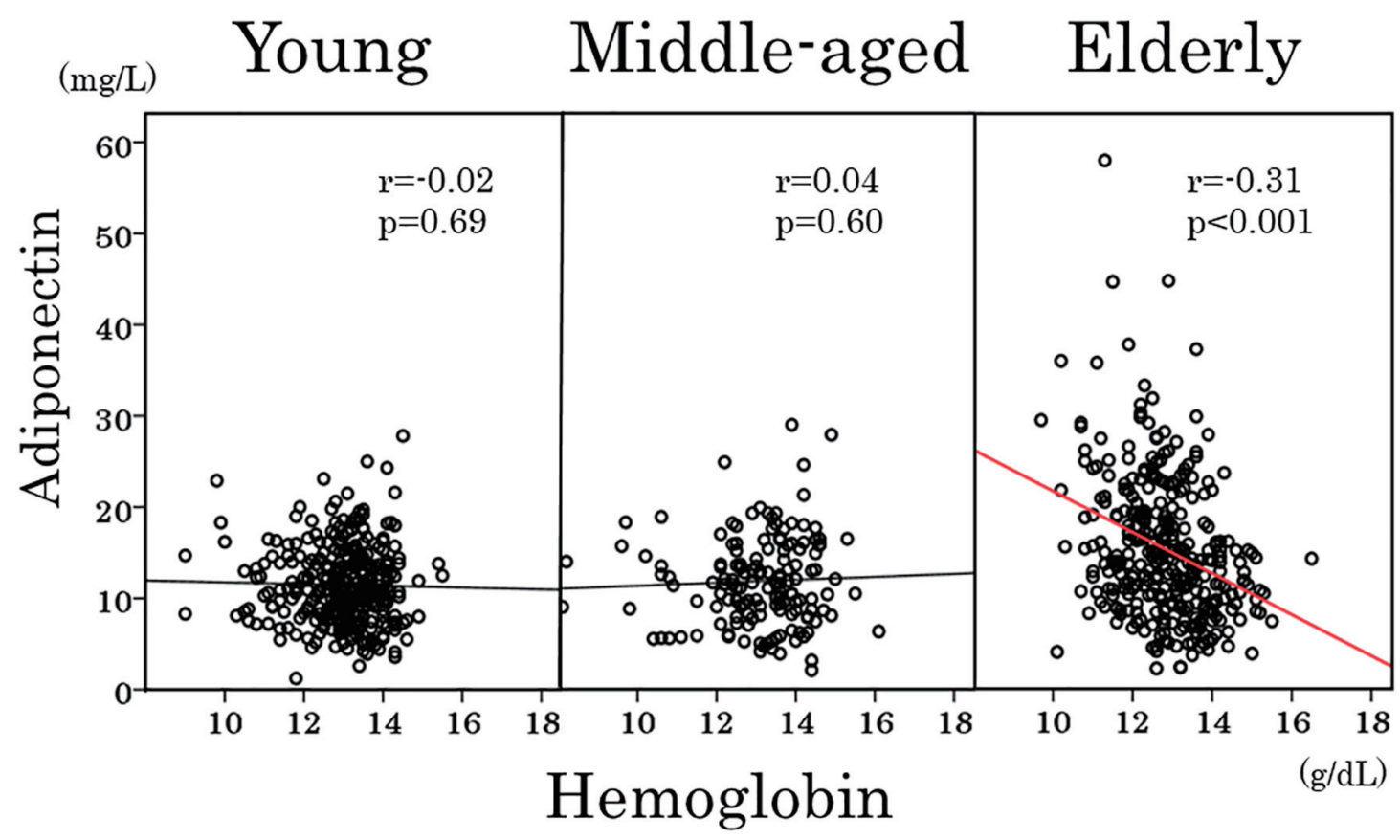

Figure 3. Scatter plots between serum adiponectin and hemoglobin in young, middle-aged and elderly women. 


\section{Young+Middle-aged Elderly}

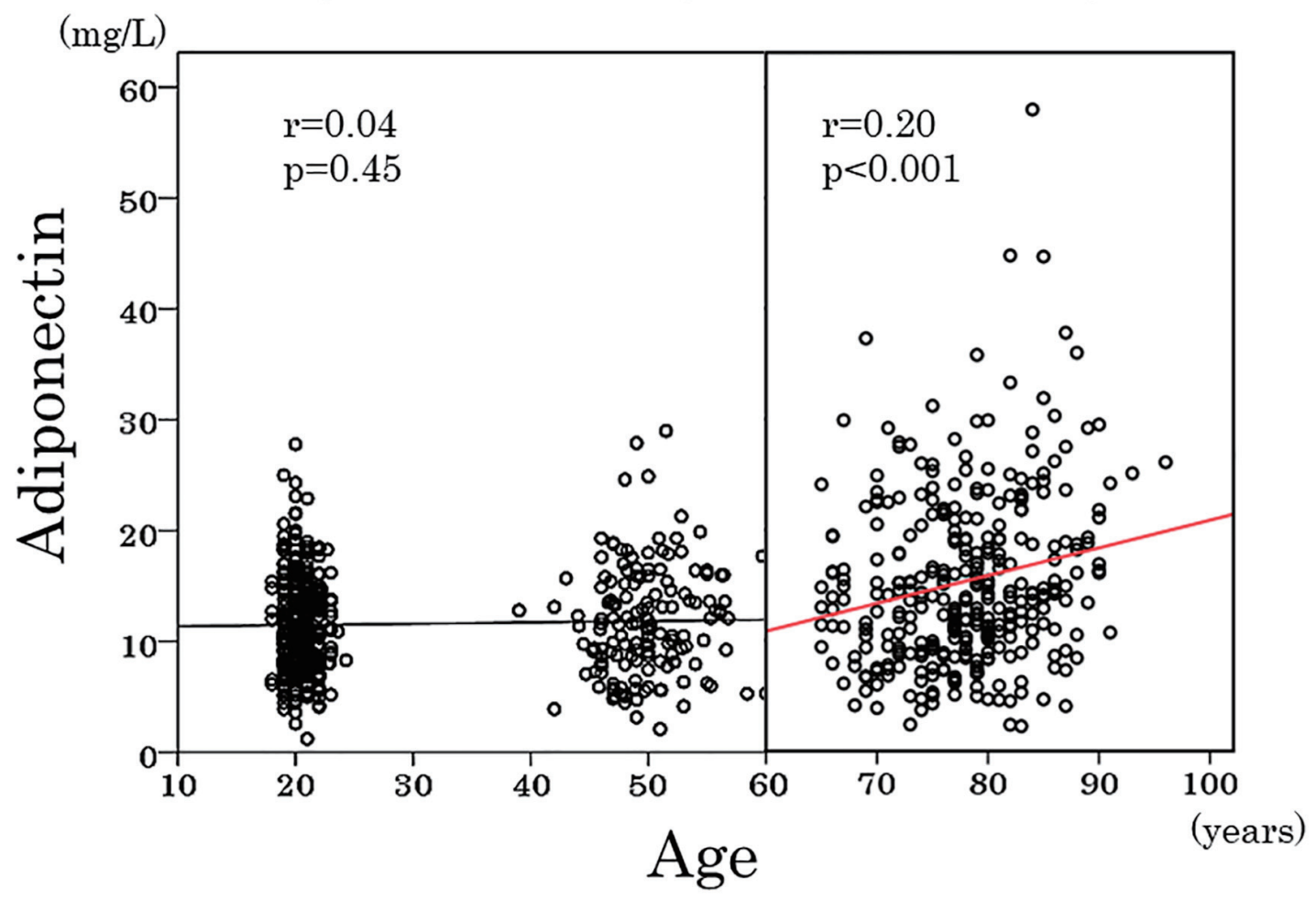

Figure 4. Scatter plots between serum adiponectin and age in young, middle-aged and elderly women. Young and middle-aged women were combined and were analyzed together.

peradiponectinemia may be a compensatory phenomenon for low hemoglobin. Another explanation is that elevated serum adiponectin may reflect accelerated adiponectin secretion in response to adverse processes or homeostatic dysregulation that accompany aging [24], that is anemia in the present study. Other age-related homeostatic dysregulation includes weight loss and physical decline [24], low muscle mass and quality
[30], heart failure [31] and renal insufficiency [32].

The present study has strengths and limitations which are similar to our previous studies [17, 22, 23]. Young and middleaged women are more homogeneous not only in genetic but also in non-genetic perspective as compared with general population. They are first-degree relatives who share about $50 \%$ of their genes. Furthermore, majority of middle-aged women

Table 4. Characteristics of Elderly Japanese Women With Hyperadiponectinemia

\begin{tabular}{llll}
\hline & & Hyperadiponectinemia & P values \\
\cline { 2 - 4 } & Yes $(\mathbf{n}=\mathbf{7 6})$ & No $(\mathbf{n}=\mathbf{2 4 6})$ & 0.014 \\
\hline Age (years) & $79 \pm 7$ & $77 \pm 6$ & $<0.001$ \\
Body mass index $\left(\mathrm{kg} / \mathrm{m}^{2}\right)$ & $20.9 \pm 2.8$ & $22.9 \pm 2.9$ & 0.18 \\
Cholesterol $(\mathrm{mg} / \mathrm{dL})$ & $222 \pm 30$ & $217 \pm 32$ & $<0.001$ \\
HDL cholesterol $(\mathrm{mg} / \mathrm{dL})$ & $75 \pm 15$ & $62 \pm 13$ & $<0.001$ \\
Leptin $(\mathrm{ng} / \mathrm{mL})$ & $5.2 \pm 3.3$ & $9.1 \pm 5.6$ & $<0.001$ \\
Adiponectin $(\mathrm{mg} / \mathrm{L})$ & $26.4 \pm 6.3$ & $11.9 \pm 4.2$ & $<0.001$ \\
Red blood cells $\left(10^{4} / \mu \mathrm{L}\right)$ & $403 \pm 34$ & $426 \pm 33$ & $<0.001$ \\
Hemoglobin $(\mathrm{g} / \mathrm{dL})$ & $12.3 \pm 1.0$ & $13.0 \pm 1.1$ & $<0.001$ \\
Hematocrit $(\%)$ & $39.5 \pm 3.1$ & $41.3 \pm 3.0$ & 0.014 \\
Anemia $(\mathrm{n}, \%)$ & $24(31.6 \%)$ & $45(18.4 \%)$ & \\
\hline
\end{tabular}

Mean \pm SD or $n, \%$. HDL: high-density lipoprotein; SD: standard deviation. 
were premenopausal. It is well known that menopause has substantial effects on body composition [33]. Limitations include the cross-sectional design that did not allow causal relationship. The recruitment procedure may also have some potential impact on the results. As the participation was voluntary, women who pay more attention to health may be more likely to participate. Biochemical parameters were measured only once. Finally, we did not have detailed drug information. Both thiazolidinediones and renin-angiotensin-system inhibitors had effects on adiponectin as well as hemoglobin levels [34-36].

\section{Conclusions}

The prevalence of hyperadiponectinemia and serum adiponectin were increased and associated with anemia at 65 years of age and older in normal-weight Japanese women.

\section{Acknowledgments}

We are indebted to all the participants for their dedicated and conscientious collaboration.

\section{Financial Disclosure}

None of the authors have any potential financial disclosure to declare associated with this research.

\section{Conflict of Interest}

We declare that we have no conflict of interest.

\section{Informed Consent}

Written informed consents were obtained from all participants.

\section{Author Contributions}

MH, AT, SM, KK, MT, MY, MK and BW have made substantial contributions to acquisition, analysis and interpretation of data. KF has been involved in drafting the manuscript. TK has been involved in revising it critically for important intellectual content, has given final approval of the version to be published and agrees to be accountable for all aspects of the work in ensuring that questions related to the accuracy or integrity of any part of the work are appropriately investigated and resolved. All authors read and approved the final manuscript.

\section{Abbreviations}

BMI: body mass index; eGFR: estimated glomerular filtration rate; HDL: high-density lipoprotein

\section{References}

1. Turer AT, Scherer PE. Adiponectin: mechanistic insights and clinical implications. Diabetologia. 2012;55(9):23192326.

2. Hanley AJ, Bowden D, Wagenknecht LE, Balasubramanyam A, Langfeld C, Saad MF, Rotter JI, et al. Associations of adiponectin with body fat distribution and insulin sensitivity in nondiabetic Hispanics and African-Americans. J Clin Endocrinol Metab. 2007;92(7):2665-2671.

3. Degawa-Yamauchi M, Dilts JR, Bovenkerk JE, Saha C, Pratt JH, Considine RV. Lower serum adiponectin levels in African-American boys. Obes Res. 2003;11(11):13841390.

4. Faraj M, Havel PJ, Phelis S, Blank D, Sniderman AD, Cianflone K. Plasma acylation-stimulating protein, adiponectin, leptin, and ghrelin before and after weight loss induced by gastric bypass surgery in morbidly obese subjects. J Clin Endocrinol Metab. 2003;88(4):1594-1602.

5. Koh SJ, Hyun YJ, Choi SY, Chae JS, Kim JY, Park S, Ahn CM, et al. Influence of age and visceral fat area on plasma adiponectin concentrations in women with normal glucose tolerance. Clin Chim Acta. 2008;389(1-2):45-50.

6. Vilarrasa N, Vendrell J, Maravall J, Broch M, Estepa A, Megia A, Soler J, et al. Distribution and determinants of adiponectin, resistin and ghrelin in a randomly selected healthy population. Clin Endocrinol (Oxf). 2005;63(3):329-335.

7. Kruger IM, Huisman HW, Schutte AE. The relationship between adiponectin, ageing and renal function in a biethnic sample. Regul Pept. 2011;169(1-3):58-63.

8. Ryan AS, Berman DM, Nicklas BJ, Sinha M, Gingerich RL, Meneilly GS, Egan JM, et al. Plasma adiponectin and leptin levels, body composition, and glucose utilization in adult women with wide ranges of age and obesity. Diabetes Care. 2003;26(8):2383-2388.

9. Obata Y, Yamada Y, Takahi Y, Baden MY, Saisho K, Tamba S, Yamamoto K, et al. Relationship between serum adiponectin levels and age in healthy subjects and patients with type 2 diabetes. Clin Endocrinol (Oxf). 2013;79(2):204-210.

10. Matsui S, Yasui T, Keyama K, Tani A, Kato T, Uemura $\mathrm{H}$, Kuwahara $\mathrm{A}$, et al. High adiponectin level in late postmenopausal women with normal renal function. Clin Chim Acta. 2014;430:104-108.

11. Schautz B, Later W, Heller M, Peters A, Muller MJ, Bosy-Westphal A. Impact of age on leptin and adiponectin independent of adiposity. Br J Nutr. 2012;108(2):363370.

12. Jurimae J, Jurimae T. Plasma adiponectin concentration in healthy pre- and postmenopausal women: relationship with body composition, bone mineral, and metabolic variables. Am J Physiol Endocrinol Metab. 2007;293(1):E4247.

13. Adamczak M, Rzepka E, Chudek J, Wiecek A. Ageing and plasma adiponectin concentration in apparently healthy males and females. Clin Endocrinol (Oxf). 2005;62(1):114-118. 
14. Jura M, Kozak LP. Obesity and related consequences to ageing. Age (Dordr). 2016;38(1):23.

15. Kohno K, Narimatsu H, Shiono Y, Suzuki I, Kato Y, Sho $\mathrm{R}$, Otani $\mathrm{K}$, et al. High serum adiponectin level is a risk factor for anemia in Japanese men: a prospective observational study of 1,029 Japanese subjects. PLoS One. 2016;11(12):e0165511.

16. Lewerin C, Johansson H, Lerner UH, Karlsson MK, Lorentzon M, Barrett-Connor E, Smith U, et al. High serum adiponectin is associated with low blood haemoglobin in elderly men: the Swedish MrOS study. J Intern Med. 2015;278(1):68-76.

17. Tsuboi A, Watanabe M, Kazumi T, Fukuo K. Anemia and reduced renal function are independent predictors of elevated serum adiponectin in elderly women. J Atheroscler Thromb. 2013;20(6):568-574.

18. Kawamoto R, Tabara Y, Kohara K, Miki T, Kusunoki T, Takayama S, Abe M. Hemoglobin is associated with serum high molecular weight adiponectin in Japanese community-dwelling persons. J Atheroscler Thromb. 2011;18(3):182-189.

19. Matsubara M, Namioka K, Katayose S. Relationships between plasma adiponectin and blood cells, hepatopancreatic enzymes in women. Thromb Haemost. 2004;91(2):360366.

20. Kohno K, Narimatsu H, Shiono Y, Suzuki I, Kato Y, Fukao A, Kubota I, et al. Management of erythropoiesis: crosssectional study of the relationships between erythropoiesis and nutrition, physical features, and adiponectin in 3519 Japanese people. Eur J Haematol. 2014;92(4):298307.

21. Guralnik JM, Eisenstaedt RS, Ferrucci L, Klein HG, Woodman RC. Prevalence of anemia in persons 65 years and older in the United States: evidence for a high rate of unexplained anemia. Blood. 2004;104(8):2263-2268.

22. Tanaka M, Yoshida T, Bin W, Fukuo K, Kazumi T. FTO, abdominal adiposity, fasting hyperglycemia associated with elevated HbA1c in Japanese middle-aged women. J Atheroscler Thromb. 2012;19(7):633-642.

23. Kitaoka K, Takeuchi M, Tsuboi A, Minato S, Kurata M, Tanaka S, Kazumi T, et al. Increased adipose and muscle insulin sensitivity without changes in serum adiponectin in young female collegiate athletes. Metab Syndr Relat Disord. 2017;15(5):246-251.

24. Kizer JR, Arnold AM, Strotmeyer ES, Ives DG, Cushman M, Ding J, Kritchevsky SB, et al. Change in circulating adiponectin in advanced old age: determinants and impact on physical function and mortality. The Cardiovascular Health Study All Stars Study. J Gerontol A Biol Sci Med Sci. 2010;65(11):1208-1214.
25. Kizer JR, Arnold AM, Jenny NS, Cushman M, Strotmeyer ES, Ives DG, Ding J, et al. Longitudinal changes in adiponectin and inflammatory markers and relation to survival in the oldest old: the Cardiovascular Health Study All Stars study. J Gerontol A Biol Sci Med Sci. 2011;66(10):1100-1107.

26. World Health Organization. Nutritional anaemias: Report of a WHO Scientific Group. Geneva, Switzerland: World Health Organization, 1968

27. Matsuo S, Imai E, Horio M, Yasuda Y, Tomita K, Nitta $K$, Yamagata $K$, et al. Revised equations for estimated GFR from serum creatinine in Japan. Am J Kidney Dis. 2009;53(6):982-992.

28. Justesen J, Stenderup K, Ebbesen EN, Mosekilde L, Steiniche T, Kassem M. Adipocyte tissue volume in bone marrow is increased with aging and in patients with osteoporosis. Biogerontology. 2001;2(3):165-171.

29. DiMascio L, Voermans C, Uqoezwa M, Duncan A, Lu $\mathrm{D}, \mathrm{Wu}$ J, Sankar U, et al. Identification of adiponectin as a novel hemopoietic stem cell growth factor. J Immunol. 2007; 178(6):3511-3520.

30. Baker JF, Newman AB, Kanaya A, Leonard MB, Zemel $\mathrm{B}$, Miljkovic I, Long J, et al. The adiponectin paradox in the elderly: associations with body composition, physical functioning, and mortality. J Gerontol A Biol Sci Med Sci. 2019;74(2):247-253.

31. Sente T, Van Berendoncks AM, Hoymans VY, Vrints CJ. Adiponectin resistance in skeletal muscle: pathophysiological implications in chronic heart failure. J Cachexia Sarcopenia Muscle. 2016;7(3):261-274.

32. Adamczak M, Chudek J, Wiecek A. Adiponectin in patients with chronic kidney disease. Semin Dial. 2009;22(4):391-395.

33. Ho SC, Wu S, Chan SG, Sham A. Menopausal transition and changes of body composition: a prospective study in Chinese perimenopausal women. Int J Obes (Lond). 2010;34(8):1265-1274.

34. Komiya N, Hirose H, Kawabe H, Itoh H, Saito I. Effects of telmisartan therapy on metabolic profiles and serum high molecular weight (HMW)-adiponectin level in Japanese male hypertensive subjects with abdominal obesity. J Atheroscler Thromb. 2009;16(2):137-142.

35. Riera-Guardia N, Rothenbacher D. The effect of thiazolidinediones on adiponectin serum level: a meta-analysis. Diabetes Obes Metab. 2008;10(5):367-375.

36. Raptis AE, Bacharaki D, Mazioti M, Marathias KP, Markakis KP, Raptis SA, Dimitriadis GD, et al. Anemia due to coadministration of renin-angiotensin-system inhibitors and PPAR $\gamma$ agonists in uncomplicated diabetic patients. Exp Clin Endocrinol Diabetes. 2012;120:416-419. 